\title{
EDITORIAL
}

\section{Achievements and advances of the Instituto de Investigaciones en Ciencias de la Salud of the National University of Asuncion, Paraguay. Year 2014}

The Instituto de Investigaciones en Ciencias de la Salud (IICS in Spanish), that depends on the Office of Rector of the National University of Asuncion, is one of the leading institutions in the areas of Research, Specialized Service and Training of Researchers through its Master Program in the health area.

In 2014 two groups of researchers from the IICS had important achievements in the area of Health Sciences. The first one was obtained as a result of the study "Distribution of VPH-16 variant isolates in Paraguayan women with different degrees of cervical lesion". This study was awarded the $\mathbf{2 0 1 4}$ National Science Prize by the Paraguayan National Congress.

The awarded work was a multidisciplinary study that started in 2007 with professionals from different areas including collaboration from scientists from Argentina and Uruguay. Among the most important findings, there is a high frequency of the high oncogenic risk Human Papilloma Virus (HPV) in women without any lesions of the uterine cervix. Most of these women being 30 years old, which is relevant because at this age a woman has more risk of having a persistent infection that with time could produce a lesion in the uterine cervix. The finding of alarming rates of women infected by HPV, revealed by this study, was possible by using a highly sensitive and specific detection method. Thus, it is possible to obtain a clear view of the disease in Paraguay.

The second achievement was obtained through the study "Diagnostic innovation applied to cases of severe acute respiratory infections in children" which was awarded an Honorable Mention of the 2014 National Science Prize. This multi-centric study, which started in 2010, allowed the quali-quantitative detection of multiple microbiological agents in hospitalized patients with severe acute respiratory infections.

The results of these works will act as a basis for the decision-makers at the level of public health to design and implement preventive and therapeutic strategies and thus, prioritize the diagnostic efforts.

All these achievements were obtained by the commitment not only from the researchers but also from the authorities of the IICS, because although it is true that the most important factor to research is the attitude and training in methodological aspects, the sustainability of the process needs the institutional economic and logistic support.

The abstracts of the awarded works are presented in the section "Others" of this number.

Other advances and achievements obtained by the IICS in this period are related to the financial awarding of 23 research projects within the frame of "Component I: Encouragement of Scientific Research of the Paraguayan Program for the Development of Science and Technology- PROCIENCIA - Call 2013". 
The awarded projects are within the frame of the following research lines of the IICS:

1. Virulence factors of virus and bacteria

2. Vector-borne diseases

3. Development and implementation of diagnostic tools

4. Health technical innovations

5. Genetic pathologies

6. Emerging pathogens

7. Environment and health

8. Autoimmune diseases

9. Prevention of uterine cervix cancer and HPV

The list of the awarded projects is presented in the section "Others" of this number.

Additionally, the Masters Program in Biomedical Sciences of the IICS was awarded financial support within the frame of the "Program of Support to the Training of Teaching - Researchers".

Besides the resources of these above mentioned projects, it will allow the training of young researchers in the research lines above mentioned and will allow them to have the option of initiating within the frame of the Masters Program in Biomedical Sciences offered by the institution.

These valuable achievements encourage the researchers of this institution to continue contributing to the development of the country and thus improve the quality of life of our population.

Prof. Dr. Nelson Apuril Cespedes, General Director of the Instituto de Investigaciones en Ciencias de la Salud, National University of Asuncion 